\title{
Visual Blood Loss Estimation Accuracy: Directions for Future Research Based on a Systematic Literature Review
}

Rachel Phillips, Marc Friberg, Mattias Lantz Cronqvist, Carl-Oscar Jonson and Erik Prytz

The self-archived postprint version of this journal article is available at Linköping University Institutional Repository (DiVA):

http://urn.kb.se/resolve?urn=urn:nbn:se:liu:diva-173322

N.B.: When citing this work, cite the original publication.

Phillips, R., Friberg, M., Lantz Cronqvist, M., Jonson, C., Prytz, E., (2020), Visual Blood Loss Estimation Accuracy: Directions for Future Research Based on a Systematic Literature Review, Human Factors and Ergonomics Society Annual Meeting Proceedings, 64(1), 1411-1415.

https://doi.org/10.1177/1071181320641337

Original publication available at:

https://doi.org/10.1177/1071181320641337

Copyright: Human Factors and Ergonomics Society

http://www.hfes.org/ 


\title{
Visual Blood Loss Estimation Accuracy: Directions for Future Research Based on a Systematic Literature Review
}

\author{
Rachel Phillips ${ }^{1}$, Marc Friberg ${ }^{2,3}$, Mattias Lantz Cronqvist ${ }^{2}$, Carl-Oscar Jonson ${ }^{3}$, and Erik Prytz ${ }^{2,3}$ \\ ${ }^{1}$ Department of Psychology, Old Dominion University. \\ ${ }^{2}$ Department of Computer and Information Science, Linköping University. \\ ${ }^{3}$ Center for Disaster Medicine and Traumatology, and Department of Biomedical and Clinical Sciences, \\ Linköping University.
}

Visual blood loss estimation occurs in a variety of medical contexts and may impact everything from interventions by immediate responders to the likelihood of receiving blood transfusions in a hospital setting. However, research suggests that visual blood loss estimation is inaccurate for laypeople and medical professionals. The aim of the current study was to conduct a systematic literature review to determine the current state of knowledge on visual blood loss estimation accuracy and identify directions for future research. A structured search resulted in 1799 titles that were subsequently screened. A total of 72 articles were coded for comparison. Based on the evaluation, several gaps were identified, most notably related to factors of the situation that may influence estimation accuracy such as blood flow and victim/patient gender. Directions for future research are proposed based on identified gaps.

\section{INTRODUCTION}

Blood loss resulting from trauma, childbirth, or a surgical procedure can be life-threatening for the patient depending on the severity of the bleeding. Understanding how much blood a trauma victim or patient has lost, and is currently losing, is therefore of great clinical importance. One simple and oftenemployed first method of estimating blood loss is to examine the source of the bleeding and the amount of blood present at the scene. For example, the Prehospital Trauma Life Support (PHTLS) course teaches students to identify "significant external hemorrhage" and "rapidly scan the patient for any sign of severe bleeding" (National Association of Emergency Medical Technicians, NAEMT, 2020). Visual blood loss estimation therefore occurs in a variety of medical contexts and may impact everything from interventions initiated by immediate responders at an accident scene to the likelihood of receiving blood transfusions in a hospital setting.

However, a plethora of research suggests that visual blood loss estimation is inaccurate and unreliable (e.g., Adkins et al., 2014; Beer et al., 2005; Bose et al., 2006; Glover, 2003; Legendre et al., 2016; Roston et al., 2012). Blood loss can be grossly over- or underestimated by both laypeople and medical professionals. As this is a known problem, prehospital care providers and medical professionals complement visual blood loss estimation with additional blood loss indicators such as vital signs and other clinical signs that the patient's body is trying to compensate. However, vital signs may not be an accurate indicator of blood loss either (Little et al., 1995) and there is often a time lag between blood loss and clinical sign manifestation. Even more accurate blood loss estimation techniques are labor-intensive, time-consuming, and require specialized equipment, such as the gravimetric method (weighing the blood containing items) or app-based programs that require taking pictures of blood containing items.

Therefore, obtaining additional measures to determine blood loss may not be feasible or available. For example, untrained laypersons lack the knowledge to use vital signs in their evaluation of injury, even if they had access to equipment. Additionally, in some situations, there may be less than five minutes to stop a life-threatening bleeding (Tjardes \& Luecking, 2018), rendering secondary blood loss estimation techniques impractical. Thus, visual blood loss estimation is likely to continue to have a major impact on immediate intervention decisions and associated health-related outcomes. From both a decision-making and educational perspective then, it is necessary to understand not only when estimation errors occur, but why, and the factors that may influence estimation accuracy. To this end, we conducted a literature review with the intention of determining the current state of knowledge on visual blood loss estimation accuracy and identifying directions for future research.

\section{METHOD}

The first step in the systematic literature review process was an exploratory database search using combinations of relevant search terms such as "estimating blood loss" or "bleeding estimation" and keywords such as "training", "emergency", "accidents", "rate", "flow", "pool", and other terms deemed relevant to the topic. These searches were conducted in 33 academic databases during 2018. Articles broadly related to the topic of human ability to visually assess blood loss were included in the initial pilot data set of approximately 145 articles. These were subject to a full-text review and summarized. A structured search was conducted in February 2020 based on this data set, where six major academic databases (Scopus, PubMed, JSTOR, SAGE, PsycInfo, and ScienceDirect) were searched using the following search string: "("visual estimation" OR "visual estimate") AND ("blood loss" OR "bleed" OR "bleeding" OR "hemorrhage")". The results were screened first based on title, then abstract (which included duplication removal), and finally full text. The results of this search and the selection process is outlined in Figure 1.

The primary inclusion criterion was that the article should try to, in some way, test people's ability to make visual blood loss estimates. Publications in languages other than English 
were excluded, as were abstract-only sources and posters due to the limited information. Also excluded were articles that primarily focused on the development of new technical methods of assessing blood loss and only used visual estimates as a baseline comparison for these technologies. The final dataset included 72 articles.

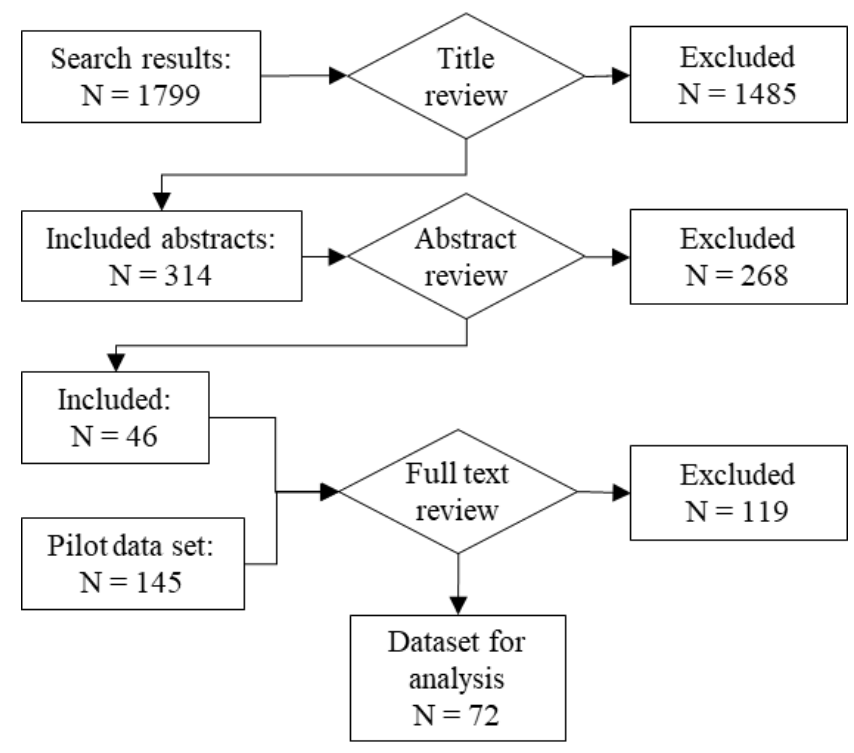

The articles in the final dataset were coded into a structured table for further analysis. The coding process was initially performed individually by four authors on subsets of the data set. The coding schemes were then compared, and a finalized coding scheme was developed based on unanimous agreement. All articles were re-coded using this coding scheme. The resultant data was used to calculate the frequency of characteristics or variables in the literature, and to identify current knowledge gaps for future research. References in the reference list that were also included in the analysis are marked with an asterisk. A complete list of references used in the analysis is available from the corresponding author by request.

\section{RESULTS}

An initial assessment of the literature was made to determine if a meta-review of statistical effects would be possible. Due to the diversity of the methods employed, the frequent lack of experimental control, and scarce reporting of statistical results it was concluded that a meta-review was not possible at this time. The literature was instead narratively summarized according to a number of relevant themes. The themes were derived from frequently occurring topics in the literature, as well as patterns derived during coding.

\section{Publication data}

A total of 72 articles were included in the analysis. Articles in the final data set were published between the years of 1967 and 2019. By decade, the 1960s, 70s, and 80s had one article each. There were seven articles published between
1990 and 1999, 23 published 2000-2009, and 39 published between 2010 and 2019 . Thus, $86.11 \%$ of the articles included in our review were published in the last 20 years. The studies reported were conducted in a variety of locations: the United States of America accounted for $33.33 \%$ of the publications, followed by the United Kingdom (16.67\%), Australia (8.33\%), France (6.94\%), and Thailand (5.56\%). There were two articles from Israel, Hong Kong, Nigeria, Sweden, and India (2.78\% each). Finally, Colombia, Singapore, Sri Lanka, Germany, Ireland, Turkey, Iran, South Africa, and Malaysia were each represented by a single article (1.39\% per location).

\section{Study populations}

The populations studied in the articles were primarily clinical professionals ( $94.44 \%$ of the articles), with an additional $4.17 \%$ incorporating medical and non-medical participants, and $1.39 \%$ exclusively examining laypersons. The most studied specialty was obstetrics $(51.39 \%)$, followed by various sub-specialties of surgery $(26.39 \%)$, emergency medicine $(15.28 \%)$, and those classified as miscellaneous or unspecified (6.94\%).

\section{Stimuli viewing conditions}

The way in which the blood loss was presented to the person, or persons, making the visual estimate differed among the articles. Of the 72 articles included in the analysis, $36.11 \%$ (26) reported studies of visual blood loss estimation associated with live medical events, specifically surgery (46.15\%) and childbirth (53.85\%). Typically, these estimates were made by clinicians present during the event and later compared to some secondary method of blood-loss estimation for an accuracy assessment. Thirty-six articles (50\%) described some form of experiment that required participants to view stimuli in person. In these, the amount of blood used to create the stimuli was most often known. Finally, 10 of the 72 included articles $(13.89 \%)$ reported studies that used photographs only $(60 \%)$, a combination of photographs and videos $(20 \%)$, or a combination of photographs and live stations $(20 \%)$.

\section{Blood stimuli}

Studies conducted during surgery and childbirth involved patient blood mixed with whatever other fluids were present in the scene. Because the studies involved live patients, the amount of blood was uncontrolled and had to be calculated for comparison against the provided estimations. The amount of blood lost was calculated using 11 different techniques including the gravimetric method, the alkaline-haematin method, and calculations based on physiological indicators.

Within the 36 articles describing studies in which participants viewed stimuli or scenarios in person, $50 \%$ used fake blood (33.33\% purchased, $44.44 \%$ homemade, and $22.22 \%$ not specified), $30.56 \%$ used expired human blood, $8.33 \%$ used human blood (whole, reconstituted, or packed red cells) but did not specify if it was expired, and $11.11 \%$ used other materials (unspecified, water, chicken, or porcine blood). All studies contained at least two separate volumes of blood, 
though the volumes themselves varied greatly, from as little as $.25 \mathrm{~mL}$ (Simpson et al., 2001) to $4000 \mathrm{~mL}$ (Roston et al., 2012).

Within the ten articles describing studies using photographs, a combination of photographs and videos, or a combination of photographs and live stations, 30\% used homemade fake blood and $60 \%$ used human blood (fresh for one, expired for the rest). There were at least five blood volume conditions in $90 \%$ of the articles, the remaining $10 \%$ only had one (Harrison \& Cooke, 1999). Volumes ranged from as little as $5 \mathrm{~mL}$ (de la Peña Silva et al., 2014) to as much as 1500mL (Parayre et al., 2015; Pranal et al., 2018).

\section{Blood flow}

Although blood flow was undoubtably present, the rate of blood flow was not mentioned as a consideration during estimation or measurement in any of the 26 studies conducted during live medical events. Blood flow was only mentioned in one of the live stations (Maslovitz et al., 2008). However, despite indicating that blood flow amount and intensity could be controlled by remote, the rate of flow was not reported or further discussed. For the studies using photographs or videos, blood flow was only present in the video conditions, and although present, the rate of flow was not specified or systematically manipulated.

\section{Surfaces and containers}

In addition to the different viewing conditions used (e.g. live stations versus photographs) the studies also differed in terms of how the blood was present in the scene. Studies conducted during surgeries or childbirth often included blood spilled on the surfaces associated with the respective procedures. This included a combination of absorbent materials (such as pads, gauze, and clothing) as well as collection devices (such as bed pans, kidney dishes, and canisters), and impervious surfaces (such as the table or hospital floor). Thus, visual blood loss estimations made in these conditions often required combining information from a variety of visual stimuli.

For the studies conducted in person using stations or simulations, there was also often a combination of conditions. Many studies incorporated conditions that involved a variety of stimuli including things like medical sponges, tapes, gauzes, pads, swabs, sheets, canisters, cups, drapes, kidney dishes, and/or bed pans. Additionally, $33.33 \%$ of the studies included blood on impervious flooring, 5.56\% included blood on concrete, and $8.33 \%$ included blood on carpet for at least one condition. Blood on clothing was included for at least one condition in $19.44 \%$ of the articles and blood in the toilet or on a sink was present for at least a condition in $11.11 \%$.

For the photograph or video-based research, blood was pictured in or on a variety of surfaces as well. Two of the experiments (20\%) used non-medical stimuli including clothing, nonabsorbent surfaces of different colors, carpet of different colors, tarmac, patient hair, and grass. Four (40\%) used only absorbent medical materials including sponges, gauze pads, and dressings and pads typically used in maternity wards, 30\% included at least one nonporous surface like the floor or mannequin, and 10\% incorporated a birthing pool.

\section{Patients or victims}

For the $53.85 \%$ of studies involving live childbirth, all patients were female. For the $46.15 \%$ of studies involving surgery, $58.33 \%$ used male and female patients, 25\% did not specify the gender of the patients, and $16.67 \%$ included only one gender due to the nature of the procedure. For the articles reporting studies based on viewing stations or simulations, a mannequin or person was present in at least one condition for $22.22 \%$ of these. In terms of patient gender, $41.67 \%$ of the studies did not include gender information. In $47.22 \%$ of the articles, the "patient" gender was either specified as female or implied to be female due to the nature of the apparatus (such as birthing pads). One scenario (2.78\%) included both genders, and three studies $(8.33 \%)$ included either a person or mannequin but the gender of these "patients" was not identified within the article. For the photograph or video-based research, $20 \%$ of the articles did not report providing gender information, $70 \%$ implied female gender, and $10 \%$ included male actors for some of the conditions.

\section{Volume estimation units and classification of accuracy}

For studies involving real cases, participants were individuals with medical training. Thus, though $46.15 \%$ did not specify the unit of measurement, they were likely measured in $\mathrm{mL}$ like the $53.85 \%$ of articles that did specify the unit of measurement. In terms of accuracy, $84.61 \%$ considered any deviation from the measured amount to be inaccurate, and half of them conducted statistical comparisons. Two studies (7.69\%) defined accuracy as within $20 \%$ of the measured volume, and $7.69 \%$ defined it as being within 400 or $500 \mathrm{ml}$.

For studies involving stations viewed in person, all but four used people with medical training as participants. Therefore, although $58.33 \%$ of the 36 articles did not specifically state the unit of measurement participants estimated in, these estimations were likely made in $\mathrm{mL}$. Twelve articles (33.33\%) specified that estimations were made in $\mathrm{mL}$, two $(5.56 \%)$ indicated that they let participants choose, and one $(2.78 \%)$ specified cubic centimeters as the unit of reporting. Of the four articles that reported patient estimations, one did not specify the unit used during estimation, one specified $\mathrm{mL}$, and the remaining two allowed participants to choose. Interestingly, the classification of accuracy also varied between studies. Fully $69.44 \%$ of the studies considered any deviation from the actual amount to be inaccurate (of these 25 articles, 28\% evaluated the statistical difference between estimated and actual blood volumes). The remaining 11 articles defined accuracy as within 50\% (2.78\%), within $20 \%$ $(19.44 \%)$, within $10 \%(2.78 \%)$, within $5 \%(2.78 \%)$, or within $50 \mathrm{~mL}(2.78 \%)$ of the actual volume.

The photograph or video-based studies all involved participants with medical training. Of the 10 articles included in our evaluation, $30 \%$ specified that the volumes were reported in $\mathrm{mL}, 20 \%$ chose from pre-defined options in $\mathrm{mL}$, and the remaining $50 \%$ provided estimations in an unspecified 
format. In terms of accuracy, any deviation from the actual volume was considered inaccurate for $70 \%$ of the articles, one of which also evaluated the differences statistically. Estimating within $20 \%$ of the actual value was considered accurate for $20 \%$ of the articles. The remaining study classified choosing the correct amount (from the provided options) or higher as accurate.

\section{DISCUSSION}

Overall, it is clear that the methods used to investigate visual estimates of blood loss are varied. Over a third of the studies were conducted during real surgeries or birth. This is beneficial as it increases the potential generalizability of results. It also indicates that the method itself is considered to be of clinical relevance, and that there are problems related to the method in natural settings. However, the findings may be hospital or situation specific and it is complicated to account for potential confounds inherent to the environment. Furthermore, the nature of live surgical or birthing events limits the ability to systematically examine factors that may be contributing to the over or underestimation of blood loss.

Over half of the studies were conducted using more controlled conditions where such factors could be systematically investigated. However, these studies were not without their limitations. A general shortcoming is the lack of theoretically guided exploration. Not a single study found in the current literature review relied on a theory of human perception or cognition in any form. Instead, the experimental designs were often based on stimuli of clinical relevance to a particular subspecialty of medical professional (e.g., the use of blood on delivery pads within obstetrics or blood on the scalp for trauma). As such, there has not been a concerted effort to systematically compare, for instance, estimation accuracy across stimuli or surface type (with two exceptions: Tall et al., 2003, and Williams \& Boyle, 2006). This is problematic as the size of a blood pool can be impacted by characteristics of the surface on which blood loss occurred (Adair \& Gallardo, 1999; Feng et al., 2018; Kreutziger et al., 2014) and estimations may be influenced by characteristics of the stimuli themselves, such as shape, size, or color. Several studies varied both volume and medium of the stimuli simultaneously, e.g. spilling $500 \mathrm{~mL}$ of blood on a cot, $25 \mathrm{~mL}$ on a 10-pack of $4 \times 4$-inch gauze, $100 \mathrm{~mL}$ on a T-shirt, and $150 \mathrm{~mL}$ poured into a "commode filled with water" (Asburn et al., 2012). Buckland and Homer (2007) used, among others, $1000 \mathrm{~mL}$ poured on sheets, $600 \mathrm{~mL}$ in a kidney dish (which had volume markings), and $350 \mathrm{~mL}$ in a bedpan and concluded that visual estimates in containers were more accurate. The few studies based on photographs or videos contained similar confounds that were not addressed and many failed to include standardized lighting or camera angles. Ultimately, this means that the extent to which the surface, blood stimulus, viewing angle, clothing, background contrast, or container shape impact blood loss estimation accuracy is not known.

Another notable gap in the current knowledge is how the flow of blood impacts visual blood loss estimations. Blood flow is one of the components used to assess the severity of surgical bleeding (Lewis et al., 2017; Spotnitz et al., 2018) and one of the criteria for determining if a trauma requires a tourniquet (Pons \& Jacobs, 2017; Goolsby et al., 2018). Yet, in the studies included in our evaluation, blood flow was only mentioned in three, as part of the procedure. During an active bleeding event, blood flow may be a prioritized part of the dynamic estimation process that needs to occur to determine the most appropriate first aid action. Unfortunately, at this time, the interaction between estimated blood flow and visual blood loss estimation is unclear. If the speed of blood flow alters the perceptions of the amount of blood (or vice versa), then educational interventions may be necessary to reduce any deleterious effects associated with this interaction.

Another potentially important variable that has not been systematically studied to date is victim or patient gender. Patient gender was either not present or not specified in over a third of the articles included in our evaluation. Additionally, no article included patient gender as a between-subjects variable when evaluating estimation accuracy. Thus, the extent to which visual blood loss estimation accuracy is impacted by victim or patient gender is unclear. Previous research has found gender related differences in prehospital care (Koval et al., 2006; Wahlin et al., 2016) and the likelihood of surviving great vessel trauma (Chen et al., 2019). If victim gender impacts visual blood loss estimation accuracy, then it will likely also impact the evaluations of injury severity and, therefore, could influence prehospital care-related decisions and subsequent treatment. Such bias, should it exist, would need to be addressed to ensure all genders were receiving equitable care at every stage post injury.

Finally, what was considered an "accurate" estimate varied across studies and the use of statistical analyses related to accuracy were limited. Most articles conclude that visual estimates are "inaccurate" to some degree (e.g., Adkins et al., 2014; Bose et al., 2006), generally an underestimate (e.g., Beer et al., 2005; Glover, 2003; Roston et al., 2012), or over or underestimate depending on volume (e.g., Legendre et al., 2016). This must, however, be interpreted in relation to the definitions of accuracy and experimental setups used in these studies. Though many articles marked any deviation from the true amount as inaccurate, others used either relative errors (such as $5,10,20$, or $50 \%$ ) or absolute errors (such as $50 \mathrm{~mL}$ or $500 \mathrm{~mL}$ ). This makes it difficult to assess the quality of the results, particularly given the lack of experimental control in about half the studies and the presence of confounds (e.g., varying both volume and medium at the same time), in many of the studies using an experimental setup.

\section{CONCLUSIONS}

The literature on visual blood loss estimations is mostly based on studies within clinical populations, with about a third featuring some form of live medical procedure and about two thirds some form of experimental setup. The experimental setups seem to have been mainly derived from clinical cases rather than guided by theory or an attempt to systematically vary potentially relevant variables that could impact blood loss estimation accuracy. The literature review therefore indicates a need for rigorous studies of accuracy and identifying sources of errors for visual blood loss estimation. 
In terms of variables, blood flow, surface or container types, laypeople populations, patient gender, and the effect of specific training interventions are some of the more relevant in need of further investigation. Blood loss volume is typically the main independent variable in past research, but the relation of volume and accuracy is still poorly understood. This is in part because of the wide variety of accuracy definitions used, and in part because of the lack of systematic experimental investigation.

Inadequate understanding of errors at the evaluation stage may result in the development of faulty intervention and training programs for laypersons or students early in their medical careers. Further experimental research would enable the development of training interventions, guides, and technology that could increase the accuracy of estimations and have a clinical impact on improving medical care both within and outside of the hospital.

\section{ACKNOWLEDGMENTS}

This study was financed in part by the Swedish Civil Contingencies Agency.

\section{REFERENCES}

Adair, T. W., \& Gallardo, A. C. (1999). Considering the target surface in bloodstain pattern analysis: An unusual case of blood pooling. Journal of Forensic Identification, 49(5), 485-493.

*Adkins, A. R., Lee, D., Woody, D. J., \& White, W. A. (2014). Accuracy of blood loss estimations among anesthesia providers. AANA Journal, 82(4), 300-306

*Ashburn, J. C., Harrison, T., Ham, J. J., \& Strote, J. (2012). Emergency physician estimation of blood loss. Western Journal of Emergency Medicine, 13(4), 376-379. doi: 10.5811/westjem.2011.9.6669

*Beer, H. L., Duvvi, S., Webb, C. J., \& Tandon, S. (2005). Blood loss estimation in epistaxis scenarios. Journal of Laryngology and Otology, 119(1), 16-18. https://doi.org/10.1258/0022215053222752

*Bose, P., Regan, F., \& Paterson-Brown, S. (2006). Improving the accuracy of estimated blood loss at obstetric haemorrhage using clinical reconstructions. BJOG: An International Journal of Obstetrics and Gynaecology, 113(8), 919-924. doi: 10.1111/j.14710528.2006.01018.x

*Buckland, S. S., \& Homer, C. S. (2007). Estimating blood loss after birth: Using simulated clinical examples. Women and Birth, 20(2), 85-88. doi:10.1016/j.wombi.2007.01.001

Chen, S., Huang, J., Tee, Y., Chen, S., Wang, S., Fu, C., Hsieh, C., \& Liao, C. (2019). Contemporary management and prognosis of great vessels trauma. Injury, 50, 1202-1207. https://doi.org/10.1016/j.injury.2019.03.054

Feng, C., Michielsen, S., \& Attinger, D. (2018). Impact of carpet construction on fluid penetration: The case of blood. Forensic Science International, 284, 184-193. https://doi.org/10.1016/j.forsciint.2018.01.009

*Glover, P. (2003). Blood loss at delivery: How accurate is your estimation? Australian Journal of Midwifery, 16(2), 21-24.

Goolsby, C., Jacobs, L., Hunt, R. C., Goralnick, E., Singletary, E. M., Levy, M. J., Goodloe, J. M., Epstein, J. L., Strauss-Riggs, K., Seitz, S. R., Krohmer, J. R., Nemeth, I., Rowe, D. W., Bradley, R. N., Gestring, M. L., \& Kirsch, T. D. (2018). Stop the Bleed Education Consortium: Education program content and delivery recommendations. Journal of Trauma and Acute Care Surgery, 84(1), 205-210.

*Harrison, J., \& Cooke, M. W. (1999). Visual assessment of blood loss by accident and emergency staff. Journal of Accident \& Emergency Medicine, 16(5), 390. https://doi.org/10.1136/emj.16.5.390-a

Koval, K. J., Tingey, C. W., \& Spratt, K. F. (2006). Are patients being transferred to level-1 trauma centers for reasons other than medical necessity? The Journal of Bone and Joint Surgery, 88(10), 2124-2132.

Kreutziger, J., Haim, A., Jonsson, K., Wenzel, V., Stark, M., \& Nussbaumer, W. (2014). Variation in size of blood puddles on different surfaces.
European Journal of Emergency Medicine, 21(5), 360-363. doi: 10.1097/MEJ.0000000000000092

*Legendre, G., Richard, M., Brun, S., Chancerel, M., Matuszewski, S., \& Sentilhes, L. (2016). Evaluation by obstetric care providers of simulated postpartum blood loss using a collector bag: A French prospective study. The Journal of Maternal-Fetal \& Neonatal Medicine, 29(21), 3575-3581.

https://doi.org/10.3109/14767058.2016.1139569

Lewis, K. M., Li, Q., Jones, D. S., Corrales, J. D., Du, H., Spiess, P. E., Menzo, E. L., \& DeAnda, A. (2017). Development and validation of an intraoperative bleeding severity scale for use in clinical studies of hemostatic agents. Surgery, 161, 771-781. http://dx.doi.org/10.1016/j.surg.2016.09.022

Little, R. A., Kirkman, E., Driscoll, P., Hanson, J., \& Mackway-Jones, K. (1995). Preventable deaths after injury: Why are the traditional vital signs poor indicators of blood loss? Journal of Accident \& Emergency Medicine, 12(1), 1-14.

*Maslovitz, S., Barkai, G., Lessing, J. B., Ziv, A., \& Many, A. (2008). Improved accuracy of postpartum blood loss estimation as assessed by simulation. Acta Obstetricia et Gynecologica Scandinavica, 87(9), 929-934. https://doi.org/10.1080/00016340802317794

National Association of Emergency Medical Technicians (NAEMT), PreHospital Trauma Life Support Committee, \& American College of Surgeons Committee on Trauma. (2020). PHTLS: Prehospital Trauma Life Support. Jones \& Bartlett Publishers.

*Parayre, I., Rivière, O., Debost-Legrand, A., Lémery, D., \& Vendittelli, F. (2015). Reliability of student midwives' visual estimate of blood loss in the immediate postpartum period: A cross-sectional study. International Journal of Nursing Studies, 52(12), 1798-1803. http://dx.doi.org/10.1016/j.ijnurstu.2015.06.015

*de la Peña Silva, A. J., Delgado, R. P., Barreto, I. Y., \& Martínez, M. D. L. P. (2014). Is visual estimation useful in determining the extent of perioperative haemorrhage? A study of correlation among anaesthetists of intermediate and high complexity hospitals in Cartagena, Colombia. Colombian Journal of Anesthesiology, 42(4), 247-254.

Pons, P. T., \& Jacobs, L. (2017). Stop the Bleed. Save a life: What everyone should know to stop bleeding after an injury. Heartford Consensus. Bleedingcontrol.org

*Pranal, M., Guttmann, A., Ouchchane, L., Parayre, I., Rivière, O., Leroux, S., ... \& Vendittelli, F. (2018). Do estimates of blood loss differ between student midwives and midwives? A multicenter crosssectional study. Midwifery, 59, 17-22. https://doi.org/10.1016/j.midw.2017.12.017

*Roston, A. B., Roston, A. L., \& Patel, A. (2012). Blood Loss: Accuracy of visual estimation. In S. Arulkumaran, M. Karoshi, L. G. Keith, A. B. Lalonde \& C. B-Lynch (Eds.) A Comprehensive Textbook of Postpartum Hemorrhage: An essential Clinical Reference for Effective Management $2^{\text {nd }}$ Edition (pp. 71-72). Sapiens Publishing.

*Simpson, R. R., Kennedy, M. L., Chew, S. B., \& Lubowski, D. Z. (2001). Do patients assess rectal bleeding accurately? ANZ Journal of Surgery, 71(11), 650-651. https://doi.org/10.1046/j.1445-1433.2001.02232.x

Spotnitz, W. D., Zielske, D., Centis, V., Hoffman, R., Gillen, D. L., Wittmann, C., ... \& McAfee, P. C. (2018). The SPOT GRADE: A new method for reproducibly quantifying surgical wound bleeding. Spine, 43(11), E664-E671. doi: 10.1097/BRS.0000000000002447

*Tall, G., Wise, D., Grove, P., \& Wilkinson, C. (2003). The accuracy of external blood loss estimation by ambulance and hospital personnel. Emergency Medicine, 15(4), 318-321.

Tjardes, T., \& Luecking, M. (2018). The platinum 5 min in TCCC: Analysis of junctional and extremity hemorrhage scenarios with a mathematical model. Military Medicine, 183(5-6), e207-e215. doi: 10.1093/milmed/usx016

Wahlin, R. R., Ponzer, S., Lövbrand, H., Skrivfars, M., Lossius, H. M., \& Castrén, M. (2016). Do male and female trauma patients receive the same prehospital care? An observational follow-up study. $B M C$ Emergency Medicine, 16(6), 1-9. doi: 10.1186/s12873-016-0070-9

*Williams, B., \& Boyle, M. (2007). Estimation of external blood loss by paramedics: Is there any point? Prehospital and Disaster Medicine, 22(6), 502-506. https://doi.org/10.1017/S1049023X0000532X 